\title{
Biomarkers and their applications in toxicology
}

\author{
Reviewed by Kamal Kishor, MSc \& Roshan Kumar Sahu, M. Pharm
}

\section{BIOMARKERS IN TOXICOLOGY,} 1ST EDN.

Edited by Ramesh C. Gupta

Academic Press, Waltham, MA

Price: $\$ 187.50$

Hardcover, 1152 pages

ISBN: 978-0-124-04649-8

Biomarkers are biochemical indicators that can be used to monitor biological changes in response to toxins or other stimuli. Researchers can measure and predict toxicological effects with the understanding that biomarkers within a biological system will fluctuate according to changes in that system. Biomarkers can help identify not only a substance's degree of toxicity but also the mechanisms by which that substance causes toxic effects. Over the last few decades, the use of biomarkers has emerged as a valuable tool in many areas of life and health sciences, so Biomarkers in Toxicology is a much-needed resource, covering descriptions and applications of biomarkers in the field of toxicology for a wide range of research purposes.

Biomarkers in Toxicology chiefly compiles recent advancements and current information about biomarkers that are used in toxicology and toxicity assessment with strong organization and clear definitions throughout the book. Over 64 chapters, Biomarkers in Toxicology reviews and discusses several categories of biomarkers, including biomarkers of experimental animal models, specific organ systems and different types of toxicity and toxicological effects. These chapters are divided into eight large parts.

Part I starts with a general overview of biomarkers and covers the basic principles, background and present role of biomarkers in toxicology research and toxicity assessments. This part includes the first eight chapters, most of which discuss different types of animal models and how each is used to assess toxicity in scientific and medical research. These models include rodents, minipigs, nonhuman primates, zebrafish and Caenorhabditis elegans. There is also a section on alternatives to animal models, and Part I concludes with a chapter on toxicokinetic-toxicodynamic modeling, which can help predict the outcomes of toxic exposures in organisms.
\end{abstract}

The next fourteen chapters comprise Part II, titled 'Systems Toxicity Biomarkers'. These chapters elaborate on biomarkers of toxicity as they appear in different organ systems. Part II discusses major physiological and anatomical systems, and the final two chapters of this part are dedicated to biomarkers of the placenta and bone marrow.

Part III and Part IV detail information about biomarkers of toxic chemicals that humans and animals encounter either incidentally or deliberately in the form of drugs and diet. Part III describes toxic chemicals that are commonly found in industrial products and our environmental surroundings; Part IV overviews biomarkers of toxicity from pharmaceuticals and nutraceuticals (dietary components that influence health and biochemical processes). Chapter 36 specifically describes nutriphenomics (how diet can interact with an animal's phenotype and affect its expression of biomarkers), how nutriphenomics can influence biomarkers in rodents, and how a researcher's choice of diet is therefore an important consideration when monitoring biomarkers. Part V covers biomarkers that arise in response to petroleum products and other chemical mixtures, which can elicit complex and synergistic reactions from biological systems.

Part VI discusses biomarkers of radiation, nanoparticles and carcinogenesis. The first chapters of this part begin with biomarkers of exposure to radiation and nanomaterials, as well as the injuries and biological responses that arise from such exposures. The following chapters then consider biomarkers of epigenetic and genetic damage before extensively discussing biomarkers of cancer and carcinogen exposure.

Part VII, titled 'Special Topics', focuses chiefly on biomarkers of diseases and pathologies, including Alzheimer's disease, Parkinson's disease, neurotoxicity, mitochondrial disruption and gross and microscopic pathological biomarkers. Part VIII, the final part, discusses applications of biomarkers in a wide range of fields spanning research, risk assessment and environmental regulation.

Biomarkers in Toxicology thoroughly conveys the very important role that biomarkers play in biological research and other applications. The 64 chapters of this book are well-organized, and readers can easily find the sections and biomarkers that are of greatest interest to them. This book introduces the background, models, causes and applications of toxicity biomarkers; it provides an indepth reference resource for those in the field of toxicology and chemical safety assessment. These topics are relevant not only to academics but also to basic scientists and researchers studying clinical applications, pharmaceuticals, pesticides, insecticides, cosmetics and other fields where toxicity is an important concern. 